\title{
PROMOÇÃO DA SAÚdE MENTAL: AÇÕES INSERIDOS NA ATENÇÃO PRIMÁRIA ${ }^{1}$
}

| Rejane Gonçalves²; Leila Pedrosa ${ }^{3}$; Márcia Oliveira ${ }^{4}$; Quenia Silva ${ }^{5}$; Renata Abreu ${ }^{6}$; Paula Pinho ${ }^{7}$

\section{RESUMO}

Contexto: A reforma psiquiátrica, apresenta, ainda, grandes desafios para a assistência da enfermagem em saúde mental comunitária, com discussão sobre a sustentabilidade do processo de mudança do modelo assistencial na prática voltada para promoção da saúde mental na atenção primária.

Objetivo: descrever as ações dos enfermeiros na promoção em saúde mental inseridos na atenção primária.

Metodologia: Estudo quantitativo, realizado com 45 enfermeiros da Microrregião do Triângulo Sul do Estado de Minas Gerais, Brasil, por meio de entrevista semiestruturada. Os dados foram analisados descritivamente e organizados em categorias.

Resultados: Dentre os 45 entrevistados, as ações realizadas para a promoção em saúde mental foram: $(84,4 \%)$ de acolhimento, $(77,8 \%)$ de orientação, $(53,3 \%)$ de atividade física, $(60,0 \%)$ de apoio familiar e $(68,9 \%)$ de educação em saúde; $(60,0 \%)$ afirmaram não possuir formação específica em saúde mental e $(68,9 \%)$ sentem-se despreparados para lidar com a saúde mental.

Conclusão: Os resultados mostraram que as concepções dos enfermeiros sobre ações de promoção da saúde mental na atenção primária estão diretamente relacionadas com a assistência e que estes profissionais reforçam a necessidade do aperfeiçoamento na área com propostas de educação permanente.

\section{PALAVRAS-CHAVE: Enfermeiros; Promoção da Saúde; Saúde Mental; Atenção Primária}

\section{RESUMEN}

Contexto: La reforma psiquiátrica, presenta grandes desafíos para la atención de enfermería en salud mental de la comunidad, con el debate sobre la sostenibilidad del cambio de la asistencia sanitaria en la práctica promoción de la salud mental en la atención primaria.

Objetivo: Describir las acciones de las enfermeras en la promoción de la salud mental en atención primaria.

Metodología: Estudio cuantitativo, realizado con 45 enfermeros Microrregião Triángulo del Sur de Minas Gerais, a través de entrevistas semi-estructuradas. Los datos fueron analizados descriptivamente y organizados en categorías.
Resultados: De los 45 encuestados, las medidas adoptadas por las enfermeras para promover la prevención y la salud mental son: $(84,4 \%)$ recepción, $(77,8 \%)$ orientación, $(53,3 \%)$ la actividad física, $(60,0 \%)$ apoyo familiar y $(68,9 \%)$ educación para la salud; $(60,0 \%)$ dijeron que no tienen formación específica en salud mental $(68,9 \%)$ no se sienten preparados para hacer frente a la salud mental.

Conclusión: Los resultados mostraron que las concepciones de las enfermeras de las acciones para promover la salud mental en atención primaria están directamente relacionadas con la asistencia profesional y refuerzan la necesidad de una mejora en la zona con propuestas para la educación continua.

\section{DESCRIPTORES: Enfermeros; Promoción de la salud; Salud Mental; Atención Primaria}

\begin{abstract}
Introduction: The psychiatric reform, also presents great challenges for nursing care in community mental health, with discussion on the sustainability of the health care change in practice focused on promoting mental health in primary care.

Objective: Describe the actions of nurses in Mental Health Promotion in primary health care.

Methods: A quantitative study, conducted with 45 nurses the Microrregião Triangle South of Minas Gerais, through semistructured interviews. Data were analyzed descriptively and organized into categories.

Results: Of the 45 respondents, the actions taken by nurses to promote prevention and mental health were: $(84.4 \%)$ reception, $(77.8 \%)$ orientation, $(53.3 \%)$ physical activity, $(60.0 \%)$ family support and $(68.9 \%)$ health education; $(60.0 \%)$ said they did not have specific training in mental health $(68.9 \%)$ feel unprepared to deal with mental health.

Conclusion: The results showed that nurses' conceptions of actions to promote mental health in primary care are directly related to professional assistance and they reinforce the need for improvement in the area with proposals for continuing education.
\end{abstract}

KEYWORDS: Nurses; Health Promotion; Mental Health; Primary Attention

1 Extraído da dissertação de Mestrado em Atenção à saúde "Ações dos enfermeiros em saúde mental na ESF”, Universidade Federal do Triângulo Mineiro, 2009.

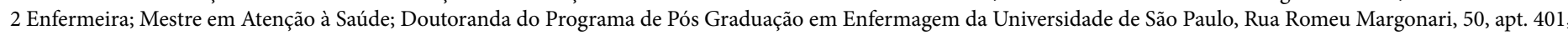
38408-072, Uberlândia/MG/Brasil, rejane.abreu@usp.br

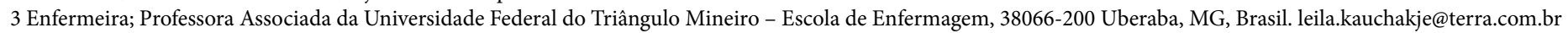
4 Enfermeira; Professora Livre-docente da Universidade de São Paulo - Escola de Enfermagem, Departamento Materno Infantil e Psiquiátrico, São Paulo, marciaap@usp.br

5 Enfermeira; Mestre em Atenção à Saúde pela Universidade Federal do Triângulo Mineiro, 38030-270, Uberaba, MG, Brasil, queniacristina21@yahoo.com.br

6 Enfermeira; Mestre em Atenção à Saúde pela Universidade Federal do Triângulo Mineiro, 38030-220, Uberaba, MG, Brasil, renata.mda@hotmail.com

7 Psicóloga; Mestre em Enfermagem Psiquiátrica; Doutoranda do Programa de Pós Graduação em Enfermagem da Universidade de São Paulo, Brasil, paulapinho@usp.br 


\section{INTRODUÇÃO}

O objeto deste estudo é as ações dos enfermeiros na promoção em saúde mental inseridos na atenção primária. Historicamente, o processo de trabalho da enfermagem existente ao longo da humanidade, mostrou-se constituído por diferentes maneiras de cuidar que, por sua vez, foram determinados pela produção de novas tecnologias influenciada, pela renovação dos saberes e evolução da ciência. O cotidiano das relações de atenção e da gestão setorial e a estruturação do cuidado à saúde devem se incorporar ao aprender e ao ensinar, formando para a saúde integral.

Com o objetivo de buscar a integralidade do cuidado à saúde, nas diversas áreas, a formação do enfermeiro deve contemplar as questões de saúde mental. Esse processo que precisa ser sustentado e valorizado consiste em responder ao sofrimento do indivíduo que busca o serviço de saúde, fornecendo um cuidado integral e atenda às necessidades do cidadão no se refere a sua saúde e não se reduza somente ao biológico (Mattos, 2001).

O conceito de Promoção da Saúde (PS) tem mudado, ao longo do tempo desde, a sua primeira utilização por Sigerist, em 1946, quando evidencia a produção social e não apenas biológica dos danos à saúde. No entanto, é no documento de 1974, o Informe de Lalonde, que se encontra uma compreensão sobre o adoecimento humano como fenômeno produzido por um conjunto de determinantes tais como: os ambientais, os sociais, o estilo de vida, a biologia humana ou a organização da atenção à saúde (Buss, 2000).

Atualmente, as bases teórico-metodológicas da PS tem sido descrita como um campo novo e um paradigma promissor na saúde, configurando sua complexidade e suas principais bases conceituais, com ênfase na integralidade do cuidado e na prevenção de agravos à saúde; o compromisso social com a qualidade de vida; e a adoção da participação com planejamento e avaliação dos processos. Tais bases favorecem o suporte à reorganização do trabalho em saúde, constituindo uma possibilidade de resposta social organizada aos problemas e necessidades de saúde de uma dada população (Chiesa et al., 2009).

Frente a isso, transformações da assistência psiquiátrica e as principais concepções de saúde e doença mental expressas nessa assistência vêm se modificando a algumas décadas, tornando o cuidado na comunidade e a desinstitucionalização do paciente psiquiátrico o foco de sua atenção. No Brasil, atualmente na área da saúde mental tem-se investido em infra-estrutura de serviços e redefinições na rede de assistência à clientela nessa área (Cardoso e Galera, 2011).
De acordo com o Ministério da Saúde [MS] (2007), a rede de saúde mental é composta por Centros de Atenção Psicossocial (CAPS), os quais são unidades de saúde que reúnem portadores de transtornos mentais de uma região e oferecem cuidados intermediários entre o regime ambulatorial e a internação hospitalar, de Serviços Residenciais Terapêuticos (moradias inseridas na comunidade) e as Unidades Psiquiátricas em Hospitais Gerais e a assistência na atenção primária. Nos CAPS, os usuários com transtorno mental são acompanhados e tratados por meio de psicoterápicos, oficinas terapêuticas e grupos operacionais com a finalidade de recuperação da saúde e reinserção social (Silva, 2011).

O Programa Saúde da Família (PSF) surge como uma estratégia de reorganização da prática assistencial com novas bases e critérios, visando assistir a família, entendida e percebida neste novo contexto, em seu ambiente físico e social, proporcionando às equipes de saúde maior abrangência na compreensão do processo saúde doença e intervenções que vão além das práticas curativas, já que o PSF converge para qualidade de vida e meio ambiente das pessoas envolvidas (Haddad, Machado, Amado e Zoboli, 2011). O processo de implantação e consolidação do Sistema Único de Saúde (SUS) e, particularmente, a reforma psiquiátrica, apresenta, ainda, grandes desafios para a assistência da enfermagem em saúde mental comunitária, com discussão sobre a sustentabilidade do processo de mudança do modelo assistencial na prática voltada para promoção da saúde mental e educação interprofissional. É nesse contexto, que surgem indagações com relação à qualidade das ações de promoção da saúde mental realizados pelos enfermeiros na atenção primária e com relação ao efeito ou impacto dessas ações junto aos portadores de transtornos mentais. Por isso, a escolha do campo da atenção primária justifica-se por representar um marco referencial brasileiro para a reorganização dos serviços de saúde, a partir da institucionalização do SUS com ações de promoção e prevenção de complicações à melhoria da qualidade de vida da população (Ferreira e Acioli, 2009). Relata-se a carência de estudos brasileiros sobre a prática em enfermagem, associada pela interação sistemática e terapêutica do cuidado, necessária a promoção da saúde mental, à resolução de problemas e a reabilitação psicossocial na atenção primária. Estudo revela que estas características tornam possível a manutenção da integridade do indivíduo, respeitada sua autonomia e liberdade. Ao desenvolver o relacionamento interpessoal na saúde mental, o enfermeiro possibilita e estimula a promoção da relação do usuário com seu corpo, seu círculo social, sua família e suas capacidades atuais (Senn, 2013). 
Considerando essas inquietações no contexto da atenção primária, e levando em consideração que o enfermeiro é o profissional referência do cuidado. Assim, o objetivo do estudo foi descrever as ações dos enfermeiros na promoção em saúde mental inseridos na atenção primária.

\section{METODOLOGIA}

Trata-se de um estudo quantitativo, que permitiu investigar com maior profundidade as ações de promoção em saúde mental realizadas pelos enfermeiros na saúde da família com os usuários portadores de sinais e sintomas de transtorno mental em uma Microrregião do Triângulo Sul do Estado de Minas Gerais (MG).

A seleção da amostra do estudo, esta realizou-se de forma não probabilística/intencional, tendo sido definidos como critérios de inclusão: os enfermeiros que fizessem parte de equipes matriciais das Unidades de Saúde da Família (USF) da referida Microrregião, por no mínimo um ano e aceitar participar da pesquisa. A amostra foi constituída por 45 enfermeiros no período de maio a dezembro de 2009.

A coleta de dados foi desenvolvida com recurso a um questionário semiestruturado elaborado pelos pesquisadores e previamente testado por um estudo piloto, composto por variáveis sociodemográficas (sexo, idade, escolaridade, tempo de formação, tempo de experiência na USF, curso de aperfeiçoamento, tipo de curso de aperfeiçoamento e experiência em saúde mental) e questões dicotômicas sobre as ações de promoção em saúde mental. A aplicação do instrumento foi realizada por meio de entrevista em ambiente privativo, na própria unidade do participante e com agendamento prévio.

O instrumento foi fundamentado nos estudos de Hildegard Peplau (1990), importantes referenciais para subsidiar a relação terapêutica entre o enfermeiro e o portador de transtorno mental e pelo Projeto Terapêutico Singular, que pode ser definido como uma estratégia de cuidado que articula um conjunto de ações resultantes da discussão e da construção coletiva de uma equipe multidisciplinar, levando em conta as necessidades, as expectativas, as crenças e o contexto social da pessoa ou do coletivo para o qual pertence (Ministério da Saúde, 2013).

Os dados obtidos foram submetidos por análises descritivas e categorizados nas quatro etapas pelo programa Statistical Package for the Social Sciencies versão 17.0 (SPSS 17.0). Em consonância com os pressupostos éticos, esse estudo foi submetido ao Comitê de Ética em Pesquisa com Seres Humanos da Universidade Federal do Triângulo Mineiro (CEP-UFTM), e aprovado sob número 1248/2008. A participação da amostra foi voluntária e todos os participantes assinaram o consentimento informado, de forma livre e esclarecida.

\section{RESULTADOS}

A análise dos dados sociodemográficos dos 45 participantes desta pesquisa identificou o seguinte perfil: 42 $(93,0 \%)$ eram do sexo feminino, com média de idade de 31,3 anos e $34(75,6 \%)$ concluíram a graduação em enfermagem nos últimos cinco anos. Com relação ao tempo de experiência na USF, verificou-se uma concentração nas faixas de 1 a 3 anos, 28 (62,2\%). Quanto à qualificação dos participantes para a saúde mental na USF, 27 (60,0\%) afirmaram não possuir formação específica em saúde mental e $35(77,8 \%)$ declararam especialistas em sua maioria, $28(62,2 \%)$ com curso de especialização em saúde da família visualizado na Tabela 1 .

TABELA 1 - Distribuição numérica e percentual dos enfermeiros na ESF de Uberaba-MG, segundo variáveis sociodemográficas, 2009

\begin{tabular}{|l|l|l|l|l|}
\hline $\begin{array}{l}\text { Variáveis sociodemográficas } \\
\text { (n=45) }\end{array}$ & $\mathbf{N}$ & $\%$ & $\begin{array}{l}\text { Média } \\
(\mathbf{d p})\end{array}$ & Mediana \\
\hline Sexo & 3 & 7,0 & & \\
Masculino & 42 & 93,0 & & \\
Feminino & & & $31,3(7,2)$ & 29,0 \\
Idade (anos) & 28 & 62 & & \\
20 a 29 & 10 & 23 & & \\
30 a 39 & 6 & 13 & & \\
40 a 49 & 1 & 2 & & \\
$>50$ & 34 & 75,6 & & \\
Tempo de enfermagem (anos) & 8 & 17,8 & & \\
1 a 5 & 2 & 4,4 & & \\
6 a 10 & 1 & 2,2 & & \\
11 a 15 & & & $3,7(2,6)$ & 3,0 \\
$>20$ & & & & \\
Tempo de experiência na USF & 28 & 62,2 & & \\
(anos) & 9 & 20,0 & & \\
1 a 3 & 7 & 15,6 & & \\
4 a 6 & 1 & 2,2 & & \\
7 a 9 & 10 & 22,2 & & \\
10 a 12 & 35 & 77,8 & & \\
Nível de aperfeiçoamento & 28 & 62,2 & & \\
Graduação & 17 & 37,8 & & \\
Especialização & 18 & 40,0 & & \\
Qualificação atual & 27 & 60,0 & & \\
Sim & & & \\
Não & & \\
Qualificação em Saúde Mental & & & \\
Sim & & \\
Não & & \\
& & \\
\end{tabular}


Entre os pesquisados, $31(68,9 \%)$ sentem-se despreparados para lidar com a saúde mental na Estratégia Saúde da Família (ESF) e $14(31,1 \%)$ declararam-se preparados. Ao serem questionados sobre o motivo pelo qual se sentem despreparados, 17 (56,1\%) dos entrevistados responderam que o motivo era inexperiência, falta de conhecimento na área, formação específica, insegurança, dificuldade na abordagem e orientação ao portador de transtorno mental; $14(43,9 \%)$ consideram que faltam cursos de capacitação, atualização periódica com ações direcionadas na saúde mental.

Dentre aqueles que responderam ter habilidades em saúde mental na USF, sete $(50,0 \%)$ consideram importante o seu preparo por meio do conhecimento, experiência, capacitação e qualificação na área; 4 (25,0\%), o contato direto com o usuário de saúde mental, por meio de ações como orientação, acolhimento, visita domiciliar, medicação e grupos de apoio; 4 (25,0\%), o tempo de trabalho na USF pode contribuir para o trabalho na área.

Dentre as ações realizadas pelos pesquisados, para a promoção em saúde mental com os usuários na atenção primária, destacou-se $38(84,4 \%)$ acolhimento, seguido de orientações $35(77,8 \%)$ e apoio familiar 27 (60,0\%). Observou-se que as ações voltadas para ações educativas em saúde mental, o genograma familiar e grupos terapêuticos não foram ressaltadas pelos enfermeiros como significativo (Tabela 2).

TABELA 2 - Distribuição numérica e percentual dos enfermeiros, segundo as ações de promoção e prevenção em saúde mental com os usuários na ESF. Uberaba-MG, 2009

\begin{tabular}{|l|l|l|}
\hline $\begin{array}{l}\text { Ações de promoção e prevenção } \\
\text { em saúde mental na USF (n=45) }\end{array}$ & $\begin{array}{l}\text { NÃO } \\
\text { N (\%) }\end{array}$ & SIM \\
N (\%) \\
\hline Acolhimento & $7(15,6)$ & $38(84,4)$ \\
Orientação & $10(22,2)$ & $35(77,8)$ \\
Apoio familiar & $18(40,0)$ & $27(60,0)$ \\
Genograma familiar & $40(88,9)$ & $5(11,1)$ \\
Grupos terapêuticos & $39(86,7)$ & $6(13,3)$ \\
Atividade física & $21(46,7)$ & $24(53,3)$ \\
Dinâmica de grupo & $31(68,9)$ & $14(31,1)$ \\
Educação em saúde & $31(68,9)$ & $14(31,1)$ \\
Nenhuma atividade & $43(95,6)$ & $2(4,4)$ \\
\hline
\end{tabular}

\section{DISCUSSÃO}

A partir dos resultados apresentados, verifica-se a contribuição do estudo para elaboração de estratégias específicas na enfermagem que favoreçam as ações em promoção da saúde mental realizadas pelos enfermeiros na atenção primária, a partir do primeiro contato com o portador de transtorno mental desde o acolhimento, propiciando o levantamento de ações que possibilitem a construção do vínculo, da relação terapêutica em um cuidado contínuo, com vistas para fornecer um suporte e/ou amenizar o sofrimento dos indivíduos e familiares, no processo de adoecimento, tratamento e na reabilitação psicossocial inserido no seu território, na família e em qualquer espaço social.

No tocante ao perfil dos participantes deste estudo é perceptível a presença prevalente do sexo feminino, jovem e na sua maioria, de recém-formados.

Em relação ao nível de aperfeiçoamento dos enfermeiros, observou-se que o percentual de enfermeiros que possuíam especialização corroborou com os dados encontrados por outros estudos (Gonçalves e Pedrosa, 2009; Rocha, Munari, Bezerra e Melo, 2009).

Em países como Portugal, os enfermeiros são generalistas e especialistas em distintas áreas de enfermagem. Nos últimos cinco anos, as escolas de enfermagem do país tem contribuído para o desenvolvimento da formação especializada e pós-graduada, com atenção para a área de saúde comunitária e familiar (Henriques, Garcia e Bacelar, 2011).

A formação específica em saúde mental pautada nos preceitos da reforma psiquiátrica e do atendimento básico de saúde ajuda a ampliar os horizontes e conduzem à reflexão no processo de humanização, solidariedade, respeito, compromisso, julgamento, aceitação, liberdade e responsabilidade.

Por outro lado, a ausência desta formação pode ser um dificultador no acompanhamento das mudanças preconizadas pelo movimento da reforma psiquiátrica (Souza, Matias, Gomes e Parente, 2007).

De acordo com os resultados obtidos, reforça-se que uma formação apropriada, com interesse, comprometimento e de qualidade em saúde mental, desde a graduação, e atualizações contínuas na área podem facilitar ações na USF, tais como acolhimento, orientação, momentos de escuta, diálogo, interação, grupos terapêuticos e dentre outras. Ações, estas que podem ser realizadas por meio de um trabalho coletivo que visa à assistência voltada para a promoção da saúde mental, propostas pela reforma psiquiátrica e pelo SUS.

A educação permanente pode estimular mudanças das práticas em saúde, fomentando a construção de ações mais inclusivas das populações em situação vulneráveis, como é o caso das famílias com pessoas com sofrimento psíquico e/ou usuárias de álcool e outras drogas (Ministério da Saúde, 2013).

Em relação aos resultados quanto as ações na promoção em saúde mental realizadas pelos enfermeiros na atenção primária, destacou-se o acolhimento ao portador de 
transtorno mental na USF, a partir do primeiro contato, como uma estratégia efetiva para a implantação dos princípios e diretrizes importantes para a prática, como a integralidade, o vínculo e a resolutividade no SUS.

Estudos evidenciaram que o acolhimento e o vínculo na atenção primária são eixos norteadores na assistência. Principalmente se desenvolvido no campo psicossocial, proporcionam aos usuários um atendimento humanizado e diferenciado em saúde. Uma estratégia que possibilita a reflexão e a fomentação de práticas humanizadas, capaz de sensibilizar os profissionais e usuários para a responsabilidade de suas ações, estabelecendo troca de saberes, construção de vínculos e qualidade dos atendimentos (Souza et al., 2007; Ferreira et al., 2009; Correia, Barros e Colvero, 2011).

Outras ações também foram mencionadas pela maioria dos participantes do estudo, como as orientações em saúde mental e o suporte familiar como estratégias que podem facilitar na promoção em saúde mental com os portadores de transtorno mental na atenção primária.

Sabendo-se que o enfermeiro tem um papel norteador voltado ao cuidado do portador de transtorno mental, destacando que o mesmo esteja preparado para acolher e apoiar as famílias no processo terapêutico, desde o início dos sinais e sintomas, diagnóstico, tratamento e ações voltadas na promoção da desinstitucionalização, já que possui as ferramentas necessárias para esse cuidado individual e à aquisição de conhecimentos a respeito do tema saúde mental, transtorno mental, relação família-usuário e reabilitação psicossocial (Borba, Paes, Guimarães, Labronici e Maftum, 2011).

A família, devido às características de proximidade e convivência, tem melhores condições de acompanhar os processos de saúde e de doença de seus membros. É fundamental buscar soluções para apoiar a família a repor o seu equilíbrio emocional. Por isso, ir-se ao encontro destas pessoas para que sejam esclarecidas as dúvidas e a expressarem o que pensam e como convivem com a situação no cotidiano, além da participação incentivada no processo do cuidar e a negociar ou adaptar novas formas para lidar com os problemas (Gomes, Martins e Amendoeira, 2011).

A criação de estratégias na atenção primária voltadas para o apoio familiar possibilita estímulo a sua participação, enquanto agente colaborador no processo terapêutico e desenvolve ações para a promoção do conforto dos portadores de transtorno mental, por meio das práticas voltadas à educação em saúde com intuito de verificar a compreensão e a adesão às informações fornecidas (Souza et al., 2007).
De acordo com os resultados, reforçam a relevância dos profissionais da saúde mental buscar incluir familiares nos projetos terapêuticos de seus integrantes portadores de transtorno mental em uma rede social. Vale mencionar que a rede social deve incluir não somente a família, como a comunidade em geral (Pinho, Oliveira, Pereira e Almeida, 2013). Portanto, o cuidado tem sido almejado, por meio da capacitação de todos os sujeitos envolvidos nesse processo (usuários, familiares, profissionais e sociedade), com a finalidade de melhor compreender a doença mental, rompendo as barreiras ao cuidado digno destas pessoas adoecidas, assegurando à qualidade da assistência à saúde mental e restaurando sua autonomia de viver em sociedade de acordo com seu potencial e os recursos disponíveis (Cardoso et al., 2011). Evidenciou-se que o profissional de saúde, em especial o enfermeiro, esteja atualizado e preparado para a prática da promoção à saúde mental, e que tenha subsídios para participar da elaboração de manuais, linhas guias e protocolos na USF, que garantam essas ações aos usuários, familiares e comunidade por meio de oficinas terapêuticas entre elas: oficinas de artesanato, de trabalhos manuais, grupos de caminhada, terapia comunitária, e, grupos abertos de acolhimento e atividades que visam discutir os aspectos relacionados ao uso de álcool e outras drogas, violência, isolamento social e entre outros. As atividades de educação em saúde e de grupos terapêuticos possibilitam a participação do enfermeiro, enquanto forma de acompanhamento e orientação de ações em saúde mental e, se expressam como estratégias que incentivam a PS, a qualidade de vida e bem-estar do ser humano (Chiesa et al., 2009). De acordo com os resultados obtidos, essas ações não foram destacadas como significantes pelos profissionais estudados. Esses dados também não foram encontrados em outros estudos nacionais como significativos na promoção das ações terapêutica dos enfermeiros em saúde mental na saúde da família.

Entende-se também que as ações de enfermagem devem ser norteadas pela educação em saúde, enquanto estratégia para a promoção da saúde da família do sujeito em sofrimento psíquico. As orientações, quando realizadas em grupo, garantem maior envolvimento dos indivíduos e familiares junto à USF, permitindo que os conhecimentos sejam mais facilmente assimilados e colocados em prática. Cabe ao enfermeiro expor conhecimento e resoluções para os problemas, disposição para ensinar, aprender e ajudar a família a encontrar meios para amenizar ou resolver seus problemas (Correia et al., 2011). 
A PS é reconhecida como importante estratégia de renovação das práticas em saúde coletiva, reconfigurando saberes e fazeres que ampliem as alternativas de qualidade de saúde e vida da população. Entretanto, as políticas e as ações de PS são ainda incipientes em relação a pouca capacidade de mudança nas práticas de atenção e de educação em enfermagem. É preciso superar os desafios e criar oportunidades para a melhoria da atenção à saúde e na qualificação do ensino. É necessário refletir a PS como um marco conceitual no redirecionamento do processo ensino-aprendizagem dos profissionais de saúde (Silva, Sena, Grillo, Horta e Prado, 2009). O cuidado do enfermeiro na atenção primária apresenta-se como uma prioridade, cada vez maior e mais complexa na ESF, estendendo-se além dos muros das unidades de saúde, procurando romper com o modelo hegemônico e fragmentado da doença mental para o modelo apoiado na promoção da saúde mental dos indivíduos, famílias, comunidades e outras instituições sociais numa perspectiva terapêutica.

Neste contexto, verificou-se a possibilidade, por meio da interação entre os profissionais da saúde da família e a equipe matricial, a promoção do diálogo entre os membros do grupo, da atualização de conhecimentos, integrando-os com a prática e o desenvolvimento de habilidades para a resolução de problemas que possam surgir obtendo, a confiança mútua entre os componentes do grupo com que se trabalha.

Estudos evidenciaram que existem avanços e inovações ao produzir uma atenção qualificada no atendimento à saúde mental com o apoio matricial. As experiências vividas pelas unidades de saúde e os profissionais pioneiros em atuar com o matriciamento revelou a necessidade de ações estruturadas para uma prática autônoma e consensual ampliada no sujeito, no território com ações individuais e coletivas necessárias para garantir o atendimento à saúde mental na atenção primária (Silva, 2011; Morais e Tanaka, 2012). Assim, a possibilidade de apoiar a inserção do enfermeiro especialista em saúde mental na equipe matricial para a capacitação, articulação com os serviços especializados, realização de ações para o planejamento, discussão dos casos clínicos com os enfermeiros generalistas e equipe interdisciplinar, devido às habilidades deste profissional para a comunicação e relacionamento interpessoal com a equipe (Soares, 2008). O cuidado clínico de enfermagem deve configurar as bases conceituais do que é a prática profissional, assim como os conceitos sobre o sofrimento psíquico e os novos paradigmas instituídos pela reforma psiquiátrica.
Para isso, novas estratégias na atenção psicossocial fazem-se necessária, como a politização dos atores, o reconhecimento dos profissionais, dos usuários do serviço e seus familiares como protagonistas na produção de sua autonomia, a partir de uma prática que envolve a dignidade, a criatividade, o acolhimento, a interdisciplinaridade, a escuta e o compartilhar de saberes (Chiesa et al., 2009).

As limitações dos resultados do estudo são inerentes ao método quantitativo, pois foi utilizada uma amostra que limita aos achados à população investigada, não sendo permitida a generalização dos dados ou de inferências a outras populações.

\section{CONCLUSÕES}

As ações de promoção em saúde mental realizadas pelos enfermeiros na atenção primária apresentaram vários elementos favoráveis no atendimento inicial, individual e na família, as quais atuaram como facilitador na relação terapêutica, como o acolhimento, as orientações e o suporte familiar. Essas ações destacaram-se como possibilidades de oferecerem aos portadores de transtorno mental que buscam sua ajuda, ações em saúde mental com foco na humanização, apoio, constituição do vínculo e do cuidado básico na atenção primária.

Porém, não evidenciado neste estudo, como significativo, as ações dos enfermeiros na promoção à saúde mental, com foco nas ações educativas e grupos terapêuticos, capazes de envolver o indivíduo na comunidade, apoiálo de acordo com suas habilidades sociais e capacidade funcional em grupo.

Os resultados apresentados reforçam a necessidade de uma política pública para a definição de intervenções e estratégias de promoção em saúde mental na atenção primária que favoreçam a consolidação das propostas de educação permanente na área, a valorização do trabalho dos enfermeiros, bem como dos outros profissionais de saúde junto aos portadores de transtornos mentais, por meio de estímulo e avaliações constantes de sua formação para prática grupal em saúde mental nos serviços primários.

Há ainda desafios setoriais que implicam na redefinição de políticas e práticas de promoção da saúde mental, com ampliação do enfoque da saúde a partir da compreensão da complexidade de mudanças sociais em curso, reiterando a relevância de se atuar não somente na desmedicalização e da reorientação das práticas de saúde, mas, sobretudo no âmbito do desenvolvimento das redes sociais, fortalecimento da cidadania, na defesa 
de políticas públicas mais efetivas, integradas e equânime. Importância de captar parcerias entre ensino/pesquisa, administração pública, organizações sociais, com vistas à efetividade e sustentabilidade das experiências e das propostas de cunho coletivo.

As limitações dos resultados do estudo são inerentes ao método quantitativo, pois foi utilizada uma amostra que limita aos achados à população investigada, não sendo permitida a generalização dos dados ou de inferências a outras populações. Os autores declaram que não há conflitos de interesse de qualquer natureza no estudo.

\section{REFERÊNCIAS BIBLIOGRÁFICAS}

Borba, L. O., Paes, M. R., Guimarães, N. A., Labronici, L. M., \& Maftum, M. A. (2011). The family and the mental disturbance carrier: dynamics and their family relationship. Revista da Escola de Enfermagem da USP, 45(2), 442-449. doi: 10.1590/S0080-62342011000200020

Buss, P. M. (2000). Promoção da saúde e qualidade de vida. Revista Ciência \& Saúde Coletiva, 5(1), 163-77.

Cardoso, L. \& Galera, S. A. F. (2011). Mental health care today. Revista da Escola de Enfermagem da USP, 45(3), 687-691. doi: 10.1590/S0080-62342011000300020

Chiesa, A. M., Fracolli, L. A., Veríssimo, M. D. L. O. R., Zoboli, E. L. C., Avila, L. K., \& Oliveira, A. A. P. (2009). Building health care technologies based on health promotion. Revista da Escola de Enfermagem da USP, 43(spe2), 1352-1357. doi: 10.1590/S008062342009000600036

Correia, V. R., Barros, S., \& Colvero, L. de A. (2011). Mental health in primary health care: practices of the family health team. Revista da Escola de Enfermagem da USP, 45(6), 1501-1506. doi: 10.1590/S008062342011000600032

Ferreira, V. A. \& Acioli, S. (2009). O cuidado na prática do enfermeiro no campo da atenção primária em saúde: produção científica. Revista Enfermagem UERJ, 17(4), 506-509.

Gomes, M. F. P., Martins, M. M., \& Amendoeira, J. (2011). As famílias com doentes mentais. Revista Portuguesa de Enfermagem de Saúde Mental (5), 52-58.

Gonçalves, R. M. D. A. \& Pedrosa, L. A. K. (2009). Perfil dos enfermeiros da estratégia saúde da família e suas habilidades para atuar na saúde mental. Ciência, Cuidado e Saúde, 8(3), 345-351. doi: 10.4025/cienccuidsaude. v8i3.9015
Haddad, J. G. V., Machado, E. P., Amado, J. N., \& Zoboli, E. L. C. P. (2011). A comunicação terapêutica na relação enfermeiro-usuário da atenção básica: um instrumento para a promoção da saúde e cidadania. O Mundo da Saúde, 35(2), 145-155.

Henriques, M. A., Garcia, E., \& Bacelar, M. (2011). The perspective of community health nursing in primary health care in Portugal. Revista da Escola de Enfermagem da USP, 45(spe2), 1786-1791. doi: 10.1590/S008062342011000800026

Mattos, R. A. (2001). Os sentidos da integralidade: algumas reflexões acerca de valores que merecem ser definidos. In Pinheiro, R. e Mattos R. A. Os sentidos da integralidade na atenção e no cuidado à saúde. (pp. 3964). Rio de Janeiro, RJ: Universidade do Estado do Rio de Janeiro.

Ministério da Saúde. (2007). Relatório de Gestão 20032006. Saúde Mental no SUS: acesso ao tratamento e mudança do modelo de atenção. Brasília (DF), Secretaria de Atenção à Saúde/DAPE.

Ministério da Saúde. (2013). Secretaria de Atenção à Saúde. Departamento de Atenção Básica. Saúde Mental. Brasília (DF), Secretaria de Atenção à Saúde/DAPE. (Cadernos de Atenção Básica, n. 34).

Morais, A. P. P. \& Tanaka, O. Y. (2012). Apoio matricial em saúde mental: alcances e limites na atenção básica. Saúde e Sociedade, 21(1), 161-170. doi: 10.1590/S010412902012000100016

Peplau, H. E. (1990). Relaciones interpersonales en enfermería. Barcelona: Salvat.

Pinho, P. H., Oliveira, M. F., Claro, H. G., Pereira, M. O., \& Almeida, M. M. (2013). A Concepção dos profissionais de Saúde acerca da Reabilitação Psicossocial nos Eixos: Morar, Rede Social e Trabalho dos Usuários de Substâncias Psicoativas. Revista Portuguesa de Enfermagem de Saúde Mental, (9), 29-35.

Rocha, B. S., Munari, D. B., Bezerra, A. L. Q., \& Melo, L. K. A. (2009). Enfermeiros coordenadores de equipe do programa saúde da família: perfil profissional. Revista Enfermagem UERJ, 17(2), 229-233.

Senn, J. F. (2013). Peplau's Theory of interpersonal relations: appication in emergency and rural nursing. Nursing Science Quarterly, 26(1), 31-35. 
Silva, D. S. (2011). Apoio matricial em saúde mental: uma análise sob ótica dos profissionais de saúde da atenção primária. Revista Portuguesa de Enfermagem de Saúde Mental (6), 20-27.

Silva, K. L., Sena, R. R., Grillo, M. J. C., Horta, N. C., \& Prado, P. M. C. (2009). Nursing education and the challenges for health promotion. Revista Brasileira de Enfermagem, 62(1), 86-91. doi: 10.1590/S003471672009000100013
Soares, M. H. (2008). A inserção do enfermeiro psiquiátrico na equipe de apoio matricial em saúde mental. SMAD, Revista Eletrônica Saúde Mental Álcool e Drogas, 4(2), [cerca de 12p]. Acedido em: http://www. revistasusp.sibi.usp.br/pdf/smad/v4n2/v4n2a06.pdf

Souza, A. J. F., Matias, G. N., Gomes, K. F. A., \& Parente, A. C. M. (2007). A saúde mental no Programa de Saúde da Família. Revista Brasileira de Enfermagem, 60(4), 391-395. doi: 10.1590/S0034-71672007000400006.

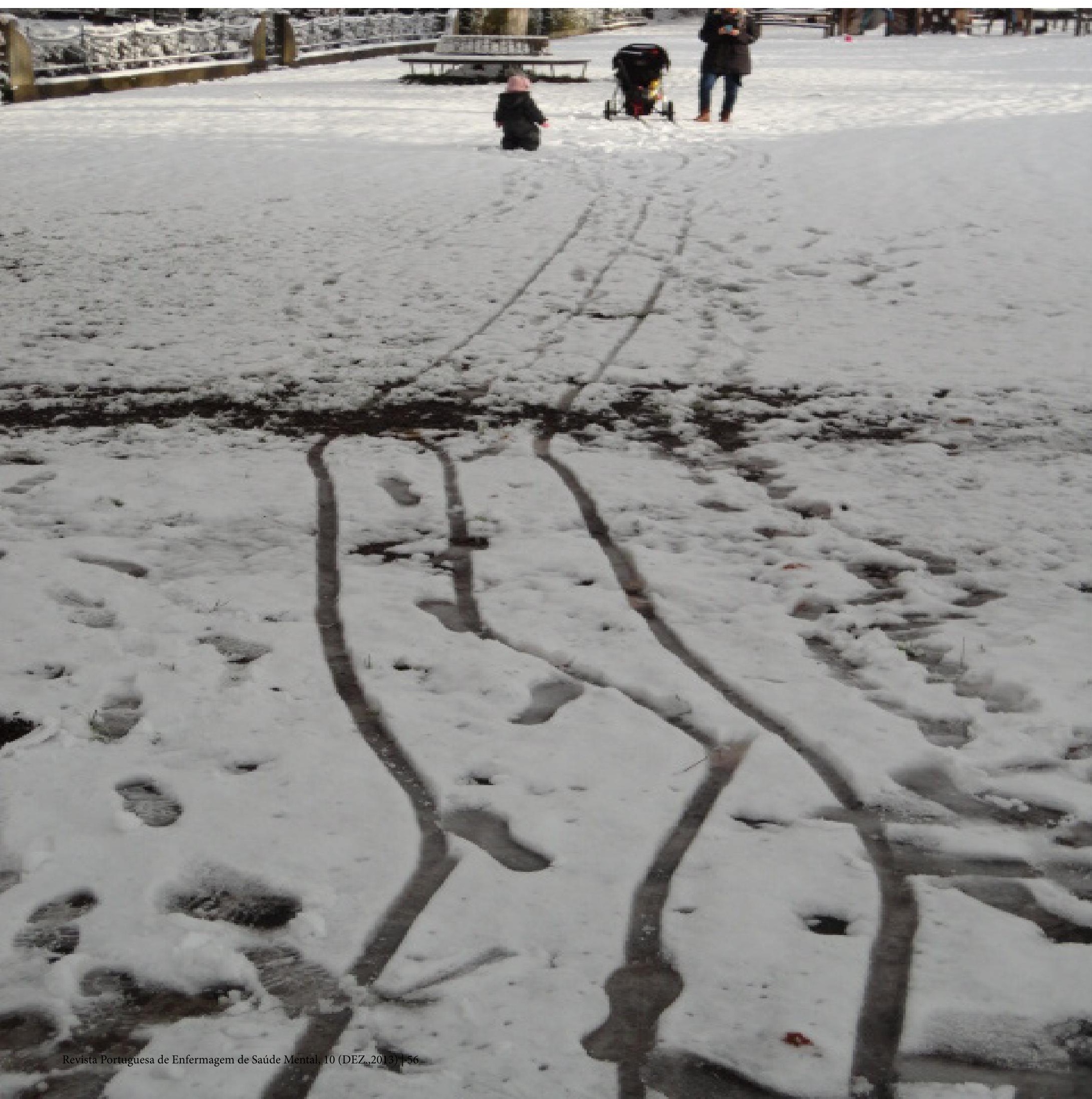

